\title{
Current Role of Surgery in Endometriosis; Indications and Progress
}

\author{
Kulvinder Kochar Kaur ${ }^{1}$, Gautam Allahbadia ${ }^{2}$ and Mandeep Singh ${ }^{3}$ \\ ${ }^{1}$ Scientific Director, Dr Kulvinder Kaur Centre for Human Reproduction, India \\ ${ }^{2}$ Scientific Director, Rotunda-A Centre for Human reproduction, India \\ ${ }^{3}$ Consultant Neurologist, Swami Satyanand Hospital, India \\ *Corresponding author: Kulvinder Kochar Kaur, Scientific Director, Dr Kulvinder Kaur Centre for Human Reproduction, India
}

Submission: April 26, 2018; Published: May 09, 2018

\begin{abstract}
Endometriosis is a chronic debilitating disease , which affects women of reproductive age group, although medical therapy may be helpful in managing pain associated with Endometriosis or infertility, surgery becomes an integral part of managing this disease .Although initially surgery was limited to $\mathrm{l}$ aporotomy associated with ovarian cystectomy and/or TAH with BSO. Laporoscopy gradually replaced that. Though diagnostic laparoscopy is used for confirmation of endometriosis by histological examination, it is not acceptable that Laporoscopy be done in multiple steps, initially to diagnose and later for treatment. Recently a lot of advancement has come in the imaging techniques by which one can combine planning of surgery based on the imaging classification. Deep endometriosis involving bowel, genitourinary tract can be dealt by careful dissection in controlled trained hands, in a well equipped set up to achieve the optimum results.Endometriosis associated infertility may or may not warrant surgery as with multiple studies operation on ovarian endometriomas might land up in reducing ovarian reserve - while doing straight IVF may result in better pregnancy rates, getting good oocyte retrieval in contrast to poor ovarian reserve resulting from damage to ovarian morphology. Use of laser for ovarian cystectomy helps in getting better outcomes than simple drainage and coagulation procedures. Robotic surgery is the latest addition, which aids in better dissection and management but its problem is its cost, not accessible to many patients and not many trained personnel available.
\end{abstract}

Keywords: Laparoscopic surgery; Ovarian endometrioma; Endometriosis associated pelvic pain; Ovarian reserve; Deep endometriosis

\section{Introduction}

Endometriosis is estimated to occur in $6-10 \%$ of women of reproductive age [1], with a prevalence of $38 \%$ (range $20-50 \%$ ) in infertile women [2] and in 71-87\% of women with chronic pelvic pain [3]. In the past symptomatic moderate to severe endometriosis was most commonly treated by laparotomy with the removal of affected tissue, with or without hysterectomy and BSO. Recently many women with advanced endometriosis have been treated by a laparosciopic approach as it results in a shorter hospitalization and recovery period as compared with laporotomy. But normal laparoscopy has inherent limitations for the treatment of advanced endometriosis because of the adhesive nature of the disease, obliteration of the surgical planes, variability of surgical skills and normal mechanics of the human hand.

\section{Indications of Surgery for Endometriosis Treatment}

There has been advancement in the management of endometriosis associated pelvic pain and infertility. The medical and surgical management of choice is mainly a thing of the past and now it is recognized that one needs to integrate both for a patient presenting with endometriosis and its associated complications Still circumstance are there when surgery is required, preferred or requested for.

Hence role of surgery in endometriosis is highlighted here besides the articles on medical treatment of endometriosis, aetiopathogenesis, endometriosis associated pain and role of dienogest and GnRH - a in Em treatment described earlier [4-6].

\section{Role of surgery in diagnosis}

Need to replace diagnostic Laparoscopy with simultaneous examination cum treatment: Surgery has been considered as the gold standard for the diagnosis of endometriosis as it gives a histological assessment of excised specimens. This is supported by the absence of definitive non invasive tests for endometriosis, although there has been continuing work on this conducted internationally [7]. Hence diagnostic laparoscopy remains the ideal route for diagnosis. Diagnostic laparoscopy for diagnostic purpose 
has various limitations, which should challenge this practice.

Though the value of having a histologic tissue diagnosis cannot be argued about here are few points which debate the role of routine Diagnostic laparoscopy.

Unrecognized lesions of endometriosis: If there are lesions having an atypical appearance they may not be recognized by the surgeon or they may be so small/subtle that pathological specimen may not be retrieved. Lesions, which are deep, below adhesions, attributable to pelvic inflammation from previous surgery or infection may get missed.Also involvement of adjacent organs which include intestinal, urinary tract and deeper nerve involvement may be missed by a laparosopic evaluation.

Excised specimens: Peritoneal or deeper lesions at the time of Diagnostic laparoscopy may not be excised always or may not be possible .Causes for this include specimen getting destroyed by crush or thermal injury at attempted removal and lack of skill to excise relevant disease areas.

Risks of surgery: The complications occurring in diagnostic and operative gynecologic laparoscopy overall might be considered to be relatively low but still they are important considerations in deciding role of surgery [8]. Various factors determine these namely experience of the surgeon, patients history and co-morbidities and the extent of disease are factors which determine the risk of complications.

To optimize patient's outcome and to minimize exposure to multiple surgeries, at present role of surgery would ideally be reserved for diagnostic confirmation and simultaneous treatment. The benefits of an examine and treat approach give the women the opportunity to confirm the pathology and address the underlying condition, all during one anesthesia. In ideal situation a single accurate surgery would also occur in the proper surgical setting with an experienced surgical team, with the correct equipment, time and assistance for the level of disease expected. Although there are always going to be exceptions to the ideal setting (i.e. unexpected finding) which would cause halting a procedure and further planning, one should strive for optimal surgical management based on a robust preoperative evaluation.

\section{Role of Surgery in Endometriosis Related Pelvic Pain}

This needs an individualized approach depending on patients presenting complaint and findings on evaluation. Chronic pelvic pain is complex and involves multiple factors beyond simply a diagnosis of endometriosis [9]. Further the issue gets complicated by the pelvic pain being an abnormal exaggerated pain response from CNS (Central sensitization). One of the prominent features of chronic pelvic pain syndrome (CPPS) women with endometriosis is decreased volume and density of the grey matter in regions related to nociception like thalamus, insular cortex and cingulated cortex .In CPPS women without endometriosis, similar findings are noted in the thalamus only while in painful cases of endometriosis, no such findings are reported $[10,11]$. Thus surgery for endometriosis might be an appropriate way of treatment but should be only used if benefits obtained is much greater than the risk of surgery. Patient centered care should prioritize pain reduction and improvement of quality of life versus optimal debulking disease which may not offer these benefits, or may cause harm. It is very difficult to decide whether there are any good effects of surgery regarding endometriosis associated pelvic pain (EAPP).

Benefits of surgery for EAPP is very difficult to outline because of lack of evidence, with limited RCT, disease presentation, varying considerably like deep, ovarian, extrapelvic and superficial, besides the variations in surgical approaches and skills of the operating team $[12,13]$. Extra genital endometriosis in 60 cases showed bowel foci in $37(61.7 \%)$, while in $13(21.7 \%)$ skin, and in $7(11.7 \%)$ urinary tract along with $3(5 \%)$ having whole pelvis localization. 2 of these had aggressive malignant transformation [14]. Duffy et al. [13] carried out a Cochrane review, which said that there was only moderate quality evidence which suggests that surgical management of mild and moderate endometriosis decreases overall pain, however there is little evidence which can compare medical therapies and very indifferent reporting of adverse events occurring during surgery [13]. But, Hirch et al. [15] conducting a systematic review in 2016 found marked variations in outcome reporting endometriosis trials which prevents generalizing these outcomes [15]. Becker et al. [16] for the World endometriosis Research Foundation collaborative elaborated guidelines on basic data which should be collected for surgical endometriosis research [16]. Although not very clear in literature more quality studies being needed, still surgery has a major role in managing EAPP.

Hidaka et al. [17] investigated the usefulness and risks of radical laparoscopic removal of deep endometriosis in patients diagnosed as stage III/IV endometriosis during laparoscopic surgery. (47 consecutive patients undergoing conservative laparoscopic surgery alone (adhesiotomy and cystectomy of ovarian endometriosis but not removal of deep endometrioic lesion; non DEL removal group) and 151 consecutive patients undergoing radical laparoscopic removal of deep endometriotic lesions combined with conservative surgery (DEL removal group) were compared. Significant pain improvement was obtained in both groups, though the degree of improvement was significantly higher and the rate of recurrence was significantly lower in the DEL removal group. This addition of radical laparoscopic removal of deep endometriotic lesions combined with conservative laparoscopic surgery significantly decreases the severity of dysmenorrhea and the rate of recurrence of pelvic pain. Though this surgical procedure remains technically demanding, amount of perioperative complications and morbidity are acceptable [17].

\section{When can surgery be considered}

Patientsdeclining/not responding/having contraindications for medical treatment: Although there are advantages of medical treatment for EAPP, all patients do not respond [18]. Currently limited medical therapies remain, like hormonal suppression and on stoppage, pain symptoms return in ladies in reproductive aged group $[19,20]$. Because of side effects like irregular menstrual bleeding, headache or mood changes or incomplete response medi- 
cal treatment is not always acceptable [12]. Thus surgery is needed in women who do not want long term medical therapy, get serious side effects or there are contraindications to medical therapy.

Many surgeons come across situations like pts requesting surgery, what they feel is a failure of medical therapy. Thus one needs to counsel regarding the limitations of surgery by itself. Though there is an overall improvement in pain symptoms, risk of pain recurrence or persistence exists. Because of this repeat surgery, in women refusing medical therapy or cannot use the same might be essential. Berland et al. [21] showed that repeat surgery might have same results as primary surgery for EAPP, but have a chance of $50 \%$ recurrence of pain at 5 years, and many women may need repeated intervention [21].

\section{Acute surgical or Pain event}

Rarely patient may present as an emergency admission when diagnosis is not clear and patient may undergo emergency laporotomy for presumed adnexaltorsion, ruptured haemorrhagic ovarian cyst in a patients whose vitals are not stable. Sometimes it may be secondary to ruptured ovarian endometrioma or endometriosis might be an accidental finding for which it is important to documents these revelations and plan for future elective care be it medical or surgical.

5.2.1.Deep endometriosis: Deep invasive endometriosis is the most severe form of endometriosis involving lot of morbidity that might cause marked organ compromise which might include genitourinary tract obstruction, renal compromise or bowel obstruction. Uccela et al. [22] detailed the surgical details, long term follow up and fertility outcomes in laparscopic ureterolysis for deep endometriosis and found it a safe procedure with encouraging pregnancy rates and found satisfactory long term results. But having hydronephrosis $\geq 2$ is associated with worse outcomes [22].

Bowel Endometriosis - This occurs in 3-37\% cases of endomrtriosis. Colorectal involvement causes alterations of bowel habit like constipation, diarrhea, tenesmus and occasionally rectal bleeding. On the basis of clinical examination, diagnosis of bowel endometriosis can be made by TVS, Barium enema examination and MRI. Thus a multidisciplinary laparoscopic treatment has become the standard of care and depending on size of lesion and site of involvement full thickness disc excision or bowel resection is performed by an experienced colorectal surgeon. Anastamotic complications occur in around 1\% cases. Long term resection of severe endometriosis is good with a pregnancy rate of 50\% [23].

These invasive lesions may limit only to the pelvis but one needs to examine the extrapelvic disease according to symptoms like in catamenial pneumothorax. Though medical therapy might be effective in many cases the surgical approach needed in many situations by treatment by trained personnel in some proper centre/institution is needed [24].

Role of combining imaging, experience and surgical technique have been described increasingly $[25,26]$. Further Abrao et al.
[28] reviewed the various critical factors in managing deep endometriosis infiltrating the rectosigmoid, besides emphasizing on role of imaging and importance of discoid and segmental resection. They concluded surgery is not indicated in all patients with deep Endometriosis, but when chosen surgery remains the therapy of choice for symptomatic patients when deep lesions do not improve with a medical treatment [28]. At present there is a greater focus on advanced imaging for endometriosis and the management of deep and ovarian endometriosis, has seen a major shift in practice [25,26]. The use of imaging for helping in diagnosis and planning any kind of surgical intervention is critical to the management of women with signs and symptoms suggesting endometriosis. Exacoustos et al. [27] carried out a ultrasound mapping system to assess the accuracy of TVS in defining size and location of deep infintrating endomeriosis (DIE) with laparosocpic/histological confirmation. They accurately mapped 104 women with suspected DIE before laparoscopic surgery. This new mapping system was developed for assessing the extent of endometriosis by measuring the size and depth of lesions at the various pelvic locations. Both surgical and histological confirmation of the USG showed that depending on different location of the lesions, the accuracy of TVS ranged from 76-97\%. The lowest sensitivity (59\%) and accuracy (76\%) were obtained for TVS in the diagnosis of vaginal endometriosis, whereas greatest efficacy (97\%) was shown in detecting bladder lesion and douglas obliteration. Hence, they concluded that this mapping system is accurate for detecting the extent of DIE and may be useful for preoperative and intraoperative management of symptomatic patients with DIE [28].

Similarly Manakaya et al. [29] gave an Ultrasound based endometriosis staging system (UBESS) to predict the level of complexity of laparoscopic surgery for endometriosis. They described three stages of UBESSI-III and correlated these with the 3 levels of complexity of laparoscopic surgery for endometriosis. Finally, they concluded that UBESS needs to be utilized to provide the level of complexity of laparoscopic surgery for endometriosis. This can facilitate the triage of women with suspected endometriosis to the most appropriate surgical expertise required for laparoscopic surgery. But, they said that this UBESS needs to be validated externally in multiple centres to assess its general applicability [29]. Ferrero [30] gave an overview of deep endometriosis and surgery for pain [30]. A systematic approach in expert hands which also relies on imaging studies in experienced operator for correct planning is needed [25]. There was a frame work provided on advanced ultrasound for deep endometriosis given by Guerriro et al. [26] of the International Deep Endometriosis Analysis group given on advanced ultrasound to give a guide for better imaging in this field. Whatever the case once surgery is needed, it is best tackled in centres having correct equipment needed for evaluating ,excising and managing this complex condition.

\section{Simultaneous Management of Concomitant Disease}

If patient is having surgery say for uterine fibroids and incidental endometriosis is found some of symptoms like dysmenorrhea may 
be because of this [31]. The correctness of surgical excision in an asymptomatic case needs to be thoroughly discussed, considering surgery carefully weighing benefits to risks.

\section{Role of Robotic Surgery}

Traditional laparoscopy has gained popularity for the management of this disease but has limitations in the surgical treatment of the most difficult cases of endometriosis. With the introduction of the robotic surgical platform experience has gradually accumulated regarding the application for surgical management of deeply infiltrating endometriosis (DIE). It has been suggested that robotic platform enables more complex dissections and may be the ideal modality of the surgical management of endometriosis. As both experience and technology expand the robotic platform will be utilized by an increasing number of surgeons for increasingly complex minimally invasive pelvic surgery. The literature analyzing the actual performance in the management of D IE, however is only just manifesting. Zanoti and Abdel bedee [32] described the unique surgical challenges of the disease. They also highlighted the current data of the literature which analyzes the application of robotic surgery to the various anatomic and clinical manifestations of endometriosis and critical outcomes as they apply to the safety, efficacy and cost of the modality of the management of endometriosis [32].

\section{Role of Surgery for Endometriosis Related Infertility}

In the presence of pelvic pain mostly medical management, excepting analgesics, prevent pregnancy. Surgery may be the only option for treatment in this subgroup. Hence, thorough discussion counseling for fertility options vis a vis surgical interventions is important. On e has to balance pain symptoms with the potential risk of harm to the reproductive organs is a common problem, in the clinical atmosphere. However, in cases where pain significantly affects ones quality of life, inability to function, then priority is to help the patient resolve these issues first. In some cases benefits of surgery may get realized through improved pain control as well as improved pregnancy rates [13]. Yet if fertility issues persist after surgical management, then appropriate evaluation and intervention will be needed. In the absence of pain, is there a role of improving fertility in w omen with Endometriosis via surgical management or improving outcomes of fertility by therapies like IVF.

\section{Mild to moderate endometriosis}

There are multiple effects which can individually or collectively affect fertility like chronic inflammation, with pro inflammatory biochemical milieu pelvic adhesions, which disrupt anatomy and affect oocyte or embryo transport and decrease ovarian reserve [33]. Surgical treatment of disease may theoretically improve the environment for successful conception. The most recent Cochrane review on this topic by Duffy et al. [13], gave evidence that laparoscopic treatment of mild and moderate Endometriosis increases live birth and ongoing pregnancy rates. There is need to balance risk of surgical intervention with alternative options for enhancing fertility like ART. Repeated surgeries may benefit pain symptoms, although they cause decrease in pregnancy rates.

\section{Deep endometriosis and infertility}

A review by Somigliana and Garcia- Velasco [34] on this topic showed that many case series show very good outcomes of surgical intervention, however one must take these series in the context of their own individual centres and acknowledge the inherent bias of case series. At this point main reason for managing deep Endometriosis surgically would be to alleviate pain or visceral obstruction in expert hands. Conservative radical excision is possible to retain the uterus and ovaries and should be the goal in those wishing to conceive.

\section{Ovarian endometrioma management}

Main indication for managing an asymptomatic ovarian Endometrioma in patients with infertility is to improve access for ART. Endometrioma size, location, transvaginal access for retrieval may all be factors in determining if patient needs surgery. In a meta analyses carried out by Hamdan et al. [35] it was shown that outcome of IVF/ICSI did not differ in women who had their Endometrioma treated surgically vs no surgical treatment [36].

\section{Hydrosalpinges in association before IVF}

Because Endometrioma Disrupts anatomy, patients may develop concurrent unilateral or bilateral hydrosalpinges and the milieu of this condition diminishes IVF success rates. At present multiple studies have shown that removal or occlusion of the tubes, which does eliminate possible natural conception, improves IVF outcomes [36].

\section{Surgical management of ovarian endometrioma}

Excising an Ovarian Endometrioma in those desiring fertility or ovarian function has been shown to be effective in controlling pain and has a lower recurrence risk in contrast to that with drainage and coagulation alone [37]. Important thing is to consider the chances of damaging the ovarian reserve following Endometrioma surgery. One question which needs an answer is how much is the effect of Ovarian Endometrioma perse on the ovarian reserve just by its mere presence and how much does surgery cause this or can it be improve omit be any ovarian reserve. Various studies which Goodman et al included, demonstrated that women having Endometriomas have lower AMH levels as compared to those not having one [38]. Excising Endometrioma decreased AMH further by 1 month, although they do seem to recover by 6 months but only back to baseline. Though this was the first study which used a control group, that included patients without Endometriosis, but in cases of Endometriomas similar results were found by different workers [39-42] regarding AMH levels.

Various things which predict more effect of surgery on Ovarian Endometrioma management is related to age and presence of bilateral ovarian cysts and B/L cystectomy [43]. More harm is done by repeated surgeries on ovarian tissue [44]. The other factors when Ovarian Endometrioma is related to presence of deep Endometriosis, more so in patients having painsymptoms [25]. Thus, the surgical procedure complexity is often beyond simple excision of the endometriotic cyst and might need increased 
dissection and warrant an interdisciplinary approach. If this ovarian disease is not fully dealt with e.g. once oophorectomy is done it may cause an ovarian remnant which might need further surgical or medical management [25]. Similarly Keyhan pointed that various factors which need to be examined before proceeding with surgery or continuing expectant management, included patients symptoms, age, ovarian reserve, size and laterality of the cyst, prior surgical treatment and level of suspicion of malignancy. Most recent evidence is for proceeding directly with IVF. These, include symptomatic infertile patients, especially those who are older, those that have diminished ovarian reserve, those having bilateral Endometriomas or those that have had prior surgical treatment. Since, surgery can further diminish ovarian reserve and presence of Endometriom as per se does not appear to affect the outcome of IVF, with surgical removal not improving IVF outcomes, this needs to be the primary target. Still proper counseling of infertile patients is essential before right option is chosen [45].

\section{Criticality of Addition of Post Operative Medical Therapy}

Main role of medical therapy following fertility sparing endometriosis surgery in women with EAPP is to prevent the recurrence of symptoms and/or disease. There may not be total surgical excision when post op medical suppression acts like adjuvant treatment for symptom control. Wu et al. [46] showed that O.C's and progestin agents showed beneficial effect and there was no statistical difference among O.C's and gestrinone, mifepristone or GnRH-a groups, though O.C; had lesser side effects which were more mild, in contrast to other hormonal treatments [46]. Also use of progestin intrauterine system has been shown to be effective in long term pain control with surgery [12]. It is important to use long term medical suppression, in contrast to short intermittent courses because symptoms and disease might return instead of returned once medicine is discontinued in women of reproductive age group [47].

Endometriomas recurrence is the main problem in patients having pain and wanting to preserve ovarian function. The recurrence rate of Ovarian Endometriomas after surgical excision might be very high-as high as $50 \%$ at $5 y$ rs. Using medical therapies like combined 0.C's is effective at decreasing recurrence and related pain recurrence [46,48-50].

But using long term post operative suppression can affect future pregnancy. Thus those wanting to conceive, there is limited role of medical suppression, as not shown to cause increase in pregnancy rates, though some studies showed better implantation rates with GnRH -a before IVF [51].

Women with deep endometriosis should also receive some type of medical treatment following surgery, as those with superficial disease and ovarian Endometriomas [52].

\section{Conclusion}

Thus surgery for endometriosis will play a role though medical management is progressing. For women having EAPP and or endometriosis related infertility one needs to consider multiple factors prior to surgery. One needs to consider how patient presents, finalizing on evaluation, USG findings and assessment based on

i. Uterus and ovary assessment

ii. Assessment of site specific tenderness

iii. Assessment of ovarian mobility and pouch of douglas obliteration

iv. Assessment of the anterior, posterior and lateral compartments of the pelvis for non bowel DIE

\section{v. Assessment of anterior wall of the bowel for DIE.}

Further response to therapies, individual treatment preferences, all have a role. There needs to be a balance between extreme situations like only surgery or only medical therapy based on patient's requirement of the disease.

\section{References}

1. Giudice LC, Kao LC (2004) Endometriosis. Lancet 364(9447): 17891799.

2. Balasch J, Circus M, Faibergues F, Carmona F, Ordi J, et al. (1996) Visible and non visible endometriois at lapariscioy in fertile and infertile women and in patients with chronic peovis pain:a prospective study. Hum Reprod 11(2): 387-391.

3. Leibson CL, Good AE, Hass SL, et al. (2004) Incidence and characterization of diagnosed endometriosis in a geographically defined poulatio. Fertil Steil 82(2): 314-321.

4. Kulvinder KK, Allahbadia GN, Singh M (2016) 4:

5. Kulvinder KK, Allahbadia GN, Singh M (2017) Meeting the challenges of endometriosis associated pai-Newer options for Future and research directions. BAOJ B informatics 1:2:1:009.

6. Kulvinder KK, Allahbadia GN, Singh M (2018) Use of Dienogest or Gonadotropin Releasing Hormone Agonist with Add back hormone therapy in longterm endometriosis medical management. J Gynecol 3(1): 000151.

7. Nisenblat V, Prentice L, Bossyut PM, Arquhar C, Hull M, et al. (2016) Combination of noninvasive tests for diagnosis of endometriosis. Cochrane Data base Sys Rev 7: CD012281.

8. Chapran C, Querteu D, Bruhat MA, Madelenal P, Fernandez H, Piere F, et al. (1998) Surgical complications of diagnostic and operative gynaecological laparoscopy:aseries of 29,9566 cases. Hum Reprod 13: 867-872.

9. Senapati S, Atashroo D, Carey E, Dassel M, Tu FF (2016) Surgical interventions for chronic pelvic pain. Cuur Opin Obstet Gynecol 28: 290296.

10. Tu CH, Niddam DM, Chao HT, Chen LF, Chen YS, Wu YT ,et al. (2010) Brain morphological changes associated with cyclic menstrual pain. Pain 150(3): 462-468.

11. As-Sanie S, Harris RL, Napadow V, Kim I, Neshewat G, Kairys A, et al. (2012) Changes in the regional gray matter volume in women with chronic pelvic pain:avoxel based morphometry study. Pain 153(5): 1006-1014.

12. Brown J, Farquhar C (2014) Endometriosis: an overview of Cochrane Review. Cochrane Data base Sys Rev.

13. Duffy JM, Arambage K, Correa FJ, Olive D, Farquhar C, Garry R, et al. (2014) Laparoscopic surgery for Endometriosis. Cochrane Data base Sys Rev pp. CD011031. 
14. Pezzolla AL, Attarulo S, Forore MG, Piscitelli D, Fabiano G, Palasciano N (2014) Extregenital endometriosis. Ann Ital Chir 85(4): 341-346.

15. Hirsch M, Duffy JM, Kuznir JO. Davis CJ, Plana MN, Khan KS, et al. (2016) Variation in outcome reporting in endometriosis trials:a systematic review. Am J Obstet Gynecol 214(4): 452-464.

16. Becker CM, Laufer MR, Stratton P, Hummelshoj L, Missamer SA, Zondervan KT, et al. (2014) WORLD Endometriosis Research Foundation: Endometriosis phenome and Biobanking Harmonisation Project. I Surgical phenotype data collection in Endometriosis research. Fertil Steril 102: 1213-1223.

17. Hidaka T, Nakashima A, Hashimoto Y, Saho S (2012) Effects of laparoscopic radical surgery for deep Endometriosis on Endometriosisrelated pelvic pain. Minim Invasive Ther Allied Technol 21(5): 355-361.

18. Bedaiwy MA, Allaire C, Yong P, Alfaraj S (2016) Medical management of Endometriosis in patients with chronic pelvic pain. Semin Reprod Med.

19. Vercellini P, Buggio L, Berlanda N, Barbara G, Somigliana E, Bosani S (2016) Estrogens-Progestins and Progestins for the management of Endometriosis. Fertil Steril 106: 1552-1571.

20. Brown J, Pan A, Hart RJ (2010) Gonadotropin Releasing Hormone Analogues for pain associated with Endometriosis. Cochrane Data base Sys Rev pp. CD008475.

21. Berlanda N, Vercellini P, Fedele L (2010) The outcomes of repeat surgery for recurrent symptomatic endometriosis. Curr Opin Obstet Gynecol 22 : 320-325.

22. Uccella S, Crorni A, Casarin J, Bogani G, Pinelli C, et al. (2014) Laparoscopy for ureteral Endometriosis: surgical details, longterm follow up and fertility outcomes. Fertil Steril 102(1): 160-166.

23. Wolthius AM, Tomassetti C (2014) Multidisciplinary laparoscopic treatment for bowel endometriosis. Best Pract Res Clin Gastroenterol 28(1): 53-67.

24. Fraser MA, Agarwal S, Chen I, Singh SS (2015) Routine vs expert -guided transvaginal ultrasound in the diagnosis of Endometriosis: a retrospective review. Abdom Imaging 40: 587-594.

25. Guereiero S, Condous G, Van den Bosch T, Valentin L, Leone FP, Van Schoubroeck D, et al. (2016) Systematic approach to sonographic evaluation of the pelvis in women with suspected Endometriosis, including terms, definitions and measurements :a consensus opinion from the Deep Endometriosis Analysis(DEA) group. Ultrasound Obstet Gynecol 48: 318-332.

26. Vercellini P, Crosignani PG, Somigliana E, Berlanda N, Barbara G, Fedelle L (2009) Medical treatment for rectovaginal Endometriosis:what is the evidence? Hum Reprod 24: 2504-2514.

27. Abrao MS, Petraglia F, Falcone T, Keckstein J, Osuga Y, Chapron C (2015) Deep Endometriosis infiltrating the rectosigmoid: critical factors to consider before management. Hum Reprod Update 21: 329-339.

28. Exacoustos C, Malzoni M, Di Giovanni A, Lazzari L, Tosti C, Petraglia F, Zupi E (2014) Ultrasound mapping system for the surgical management of deep infiltrating Endometriosis. Fertil Steril 102(1): 143-150.

29. Menakaya U, Reid S, Lus C, Bassem G, Infante F, Condous G (2016) Performance of iltrasound based Endometriosis staging system(UBESS) for predicting level of complexity of laparoscopic surgery for Endometriosis. Ultrasound Obstet Gynecol 48: 786-795.

30. Ferrero S, Alessandri F, Racca A, Leone RMU (2015) Treatment of pain associated with Deep Endometriosis: alternatives and evidence. Fertil Steril 104(4): 771-792.

31. Nezhat C, Li A, Abed S, Balassiano E, Soliemannjad R, Nezat A, et al. (2016) Strong association between endometriosis and symptomatic leiomyomas. JSLS 20(3).
32.Zanotti KM, Abdelbadee AY (2015) Robotic management of Endometriosis: where do we stand? Minerva Gynecol 67(3): 257-272.

33. Vercellini P, Vigano P, Somigliana E, Fedelle L (2014) Endometriosis: Pathogenesis and treatment. Nat Rev Endocrinol 10: 261-275.

34. Somigliana E, Garcia-Velasco JA (2015) Treatment of infertility associated with deep Endometriosis: definition of therapeutic balances. Fertil Steril 104(4): 764-770.

35. Hamdan M, Dunselman G, Li TC, Cheong Y (2015) The impact of endometrioma on IVF/ICSI outcome: a systematic review and meta analysis. Hum Reprod Update 21(6): 809-825.

36. Johnson N, Van Voorst S, Sowter MC, Strandell A, Mol BW (2010) Surgical treatment for tubal disease in women due to undergo in vitro fertilization. Cochrane Data base Sys Rev 3: CD002125.

37. Beretta P, Franchi M, Ghezzi F, Bussaca M, Zupi E, Bollis P (1998) Randomized clinical trial of two laparoscopic treatments of endomtriomas: cystectomy versus drainage and coagulation. Fertil Steril 70(6): 1176-1180.

38. Goodman LR, Goldberg JM, Flyckt RL, Gupta M, Harwalker J, Falcone $\mathrm{T}$ (2016) Effect of surgery on ovarian reserve in women with endometriomas, endometriosis and controls. Am J Obstet Gynecol 215: 589.

39. Uncu G, Kasapoglu I, Ozerkan K, Seyhan A, Yilmazlepe OA, Ata B (2013) Prospective assessment of the impact of endometriomas and their removal on ovarian reserve and determinants of the rate of decline in ovarian reserve. Hum Reprod 28(8): 2140-2145.

40. Chen Y, Pei H, Chang Y, Chen M, Wang H, Xie H, et al. (2014) The impact of ovarian endometriomas and laparoscopic cystectome on ovarian reserve and the exploration of related factors assessed by serum antiMullerian hormone: a prospective cohort study. J Ovarian Res 7: 108.

41. Raffi F, Metwailly M, Amer S (2012) The impact of excision of endometriomas on ovarian reserve: a systematic review and meta analysis. J Clin Endocrinol Metab 97: 3146-3154.

42. Somigliana E, Barlanda N, Benaglia L, Vigano P, Vercellini P, Fedele L (2012) Surgical excision of endometriomas and ovarian reserve: a systematic review on serum anti-Mullerian hormone level modifications. Fertil Steril 98(6): 1531-1538.

43. Alborizi S, Keramati P, Younesi M, Samsami A, Dadras N (2014) The impact of laparoscopic cystectome on ovarian reserve in patients with unilateral and bilateral endometriomas. Fertil Steril 101(2): 427-434.

44. Muzli L, Achilli C, Lecce F, Bianchi A, Franceschetti M, Marchetti C, et al. (2015) Second surgery for recurrent endometriomas is more harmful to healthy ovarian tissue and ovarian reserve than first surgery. Fertil Steril 103(3): 738-743.

45. Keyhan S, Hughes C, Price T, Muasher S (2015) An update on surgical versus expectant management of ovarian endometriomas in infertile women. Bio Med Research International Article ID 204792.

46. Wu L, Wu Q, Liu L (2013) Oral contraceptive pills for endometriomas after corrective surgery: a systematic review and meta analysis. Gynecol Endocrinol 29: 883-890.

47. Busacca M, Somigliana E, Bianchi S, De Marinis S, Calia C, Candiani M, et al. (2001) Postoperative GnRH analogue treatment after conservative surgery for symptomatic endometiosis stage III-IV:arandomized controlled study. Hum Reprod 16(11): 2399-2402.

48. Vercellini P, de Matteis S, Somigliana E, Buggio L, Frattaruolo MP, Fedelle $\mathrm{L}$ (2013) Long term adjuvant therapy for the prevention of post operative endometrioma recurrence :a systematic review and meta analysis. Acta Obstet Gynecol Scand 92(1): 8-16. 
49. Muzli L, di Tucci C, Achilli C, Di Donato V, Musella A, Palaia I, et al. (2016) Continuous versus cyclic Oral contraceptives after laparoscopic excision of ovarian endometriomas: a systematic review and meta analysis. Am J Obstet Gynecol 214(2): 203-211.

50. Vercellini P, Somigliana E, Vigano P, de Matteis S, Barbara G, et al. (2010) Post operative endometiosis recurrence: a plea for prevention based on pathogenetic, epidemiological and clinical evidence. Rprod Biomed Online 21(2): 259-665.
51. Sallam HN, Garcia-Velasco JA, Dias S, Arici A, Abou Setta AM (2006) Long term pituitary down regulation before in vitro fertilization(IVF) for women with endometriosis. Cochrane Data base Sys Rev 1: CD004635.

52. Somigliana E, Busnelli A, Benaglia L, Vigano P, Leonardi M, et al. (2016) Postoperative hormonal therapy after surgical excision of deep endometriosis. Eur J Obstet Gynecol Reprod Biol 209: 77-80.

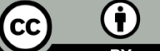

Creative Commons Attribution 4.0 International License

For possible submissions Click Here

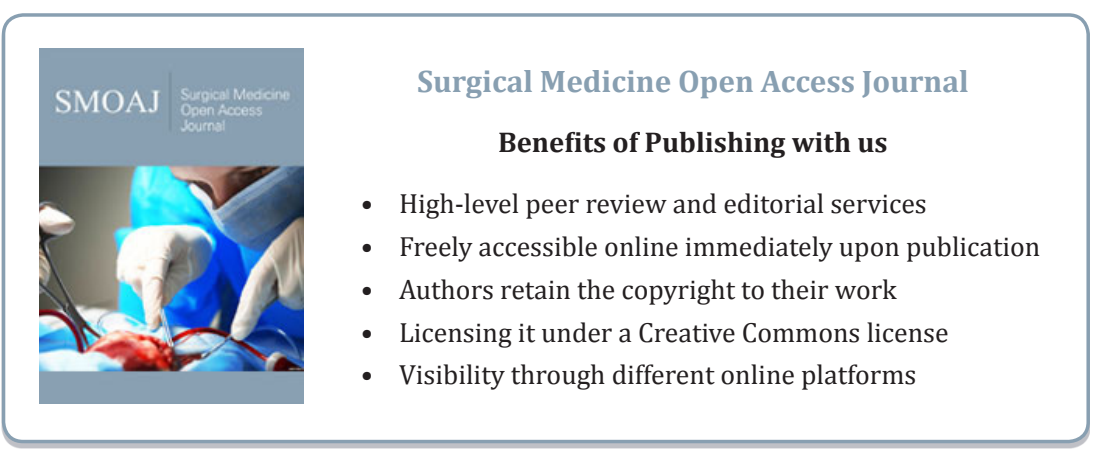

